\title{
TeXture Manipulation By ION IMPLANTATION
}

\author{
K. HELMING \\ JINR Dubna, Head Post Office P.O. Box 79, \\ 101000 MOSCOW, USSR
}

\author{
B. RAUSCHENBACH \\ CINR Rossendorf, P.O. Box 19, \\ 8051 Dresden, GDR
}

\begin{abstract}
High fluence nitrogen ion implantation into titanium at room temperature results in the formation of titanium nitride precipitation. Electron diffraction measurements reveal an implantation-induced texture of titanium nitride precipitations. The experiments demonstrate a strong coupling effect between the implantation-induced fibre texture and the direction of implantation. The results of this investigation support the assumption that the effect of implantation-induced texture is caused by the process of seed selection by ion channeling.
\end{abstract}

\section{INTRODUCTION}

The anisotropy of physical properties of polycrystalline materials depends mainly on the texture, i.e. on the orientation distribution function (ODF) of the crystallites. The ODF may be reproduced from pole figures. In general, pole figures of bulk material can be determined by X-ray or neutron diffraction. For the study of texture of small areas (some $\mu m$ ) or thin layers $(\sim 1 \mu m$ thickness), electron diffraction must be used, nevertheless the measurement of the electron intensity and its interpretation are very difficult. The texture of thin layers can be determined directly by single orientation measurement with electron diffraction ${ }^{1}$ or by pole figure measurement ${ }^{2-4}$.

It is well known, that the high-fluence ion implantation is able to improve the surface properties of metals and alloys. To optimize this effect, we have studied the alignment effect of ion implantation on crystallographic orientation. Recently, it was found that the ion bombardment has a pronouced alignment effect on crystallographic orientation of thin films ${ }^{4,9-12}$. In our earlier communication $^{4}$, we determined a preferred orientation of formed $\mathrm{TiN}$ (fcc) crystallites after nitrogen ion implantation of hexagonal titanium by electron diffraction ${ }^{9}$ and calaculated the orientation distribution funtion (ODF) from highly incomplete pole figures. In this paper a systematic study by transmission high-energy electron diffraction of the implantation-induced texture of titanium 
nitride ${ }^{5}$ is given.

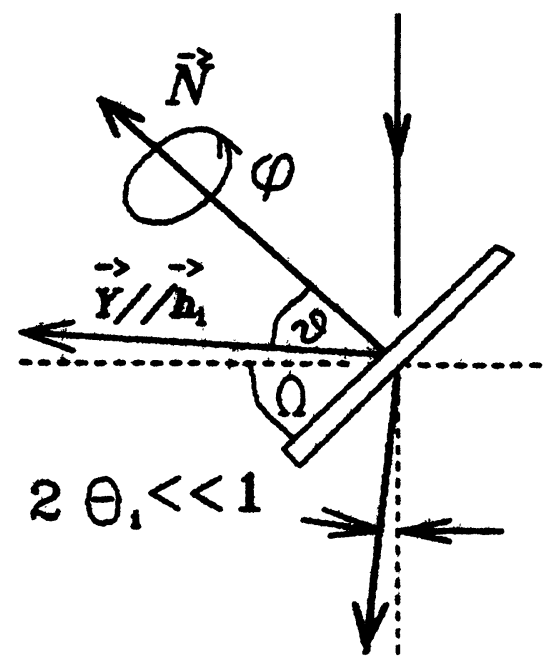

Figure 1: Schematic representation of the angle arrangement by electron diffraction on the crystal plane $\vec{h}_{i}$ of a tilted sample:

$\Theta_{i}$ - Bragg angle, $\vec{Y}$ - scattering vector, $\varphi$ - azimuth angle, $\vartheta$ - angle of tilting, $\vec{N}$ sample normal, $\Omega=90^{\circ}-\vartheta$.

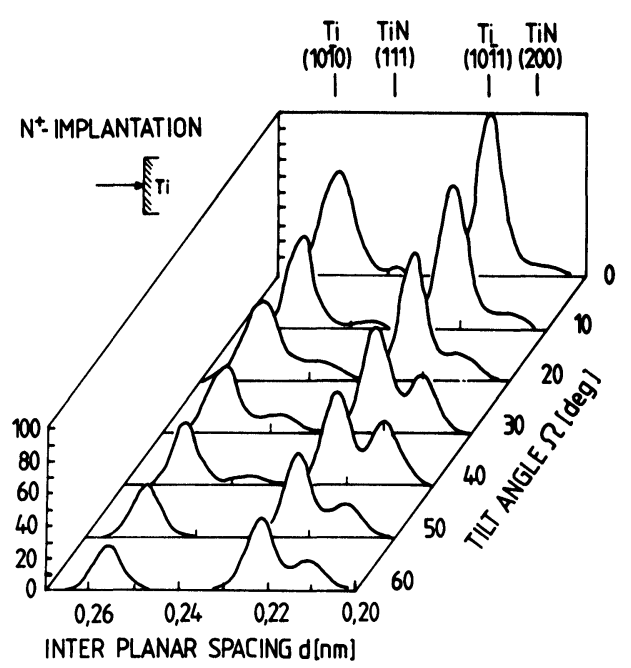

Figure 2: Diffracted and normalized Intensities of selected Tiand TiN- reflections

\section{EXPERIMENTAL CONDITIONS}

Polycrystalline layers of titanium were deposited on $\mathrm{NaCl}$ or carbon in high vacuum during electron beam evaporation. The deposition rate was monitored and controlled. The thickness of the titanium layers was about $110 \mathrm{~nm}$, i.e. $2 R_{p}$, where $R_{p}$ is the mean projected range of the nitrogen ions at the used implantation energy. The high-fluence implantation of nitrogen ions was carried out with $50 \mathrm{keV}$ ions at room temperature in a vacuum better than $1 \times 10^{-4} \mathrm{~Pa}$. Ion currents were maintained below $5 \mu \mathrm{mA} / \mathrm{cm}^{2}$ and the ion fluence was $1 \times 10^{17}, 5 \times 10^{17}$ or $1 \times 10^{18}$ ions/ $\mathrm{cm}^{2}$. This low current ion density was chosen in order to avoid heating effects during implantation. After implantation, the layer structure was investigated by the high-voltage transmission electron microscopy (HVEM) and the transmission high-energy electron diffraction (THEED) in combination with an inside goniometer. The technique of ion channeling (1.7 $\mathrm{MeV} \mathrm{He}{ }^{+}$-ions) has been used to study the lattice disorder. Some samples were annealed in high-vacuum $\left(10^{-4} \mathrm{~Pa}\right)$ at $400^{\circ} \mathrm{C}$ for $2 \mathrm{~h}$.

The measurement of pole figures base on the selected area diffraction pattem (SAD). Fig. 1 illustrates the tilting of the sample and the electron beam arrangement. From this the pole figures are obtained $\left(\Delta \vartheta=2.5^{\circ}\right.$ or $\left.5^{\circ}\right)$. The pole figures depend on the direction of the scattering vector $\vec{y}=(\vartheta, \varphi)$ in regard to the sample with the normal $\vec{N}$. Because the Bragg angle $\Theta$ is very small by 
THEED $(\ll 1), \vartheta$ is given by the angle of tilting $\Omega=90^{\circ}-\vartheta$. Since the angle of tilting $\vartheta$ may only range between $30^{\circ} \leq \vartheta \leq 90^{\circ}$, the transmission pole figures are extremly incomplete.

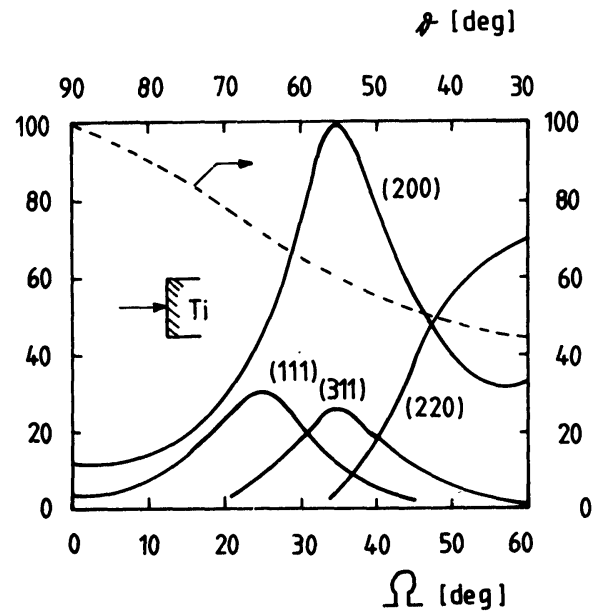

Figure 3: Diffraction pole figures $(200),(111),(220)$ and (311). In addition, the normalized intensity of the primary electron beam is also shown

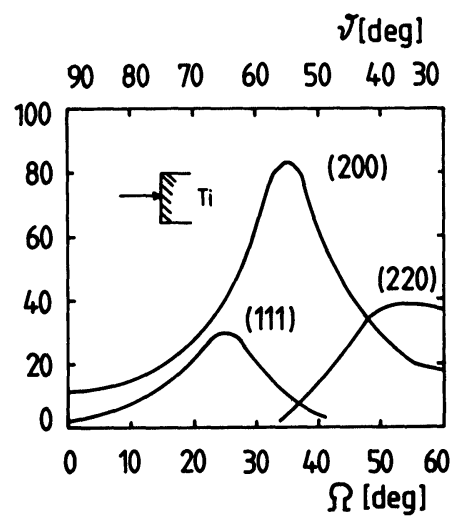

Figure 4: The reproduced pole figures (200),(111) and (220)

\section{Results}

The high-fluence nitrogen ion implantation in titanium forms nitride precipitates for fluences $>1-5 \times 10^{16}$ ions $/ \mathrm{cm}^{2} 9$. Therefore titanium was implanted with $5 \times 10^{17} \mathrm{~N}^{+}$-ions $/ \mathrm{cm}^{2}$ at room temperature. Depending on the fluence, the precipitates were identified as fcc $\delta-\mathrm{TiN}_{x}$ and the tetragonal $\epsilon-\mathrm{Ti}_{2} \mathrm{~N}$ phases (details $\mathrm{in}^{9}$ ). The fcc titanium nitride phase was formed preferentially.

The implantation induced texture of the fcc titanium nitride formed precipitates were investigated in situ using tilting experiments in the electron microscope. The thin titanium films were perpendicular implanted, i.e. in direction of the surface normal $\vec{N}$. The diaphragm setting was always chosen so that more than $10^{4}$ precipitates participated on the diffraction experiment. In order to get information on the texture, the perpendicular implanted titanium samples were tilted in small selected angular steps $\left(\Delta \Omega=2.5^{\circ}\right.$, cf. Fig. 1) and the intensities were measured. The notable features of the measurements are the followings:

(1) The intensities of the diffraction rings and also of the primary electron beam are decreased with increasing the tilt angle $\Omega$ around an axis perpendicular to the primary beam.

(2) By measurement of the intensity as function of the azimuth angle $\phi$ we found in the case $\vec{N}$ parallel to the primary beam a radial-symmetrical behaviour (fibre texture, fibre axis $\vec{C} \| \vec{N}$ ) for the whole range of the measurement, i.e. the pole figures were independent of the azimuth angle $\varphi$. In order to test the fibre texture, the peak intensities along the strongest 
Debye rings were measured for several azimuth angles. The presence of a fibre texture was also found by Harper et al. ${ }^{8}$ after ion assisted deposition.

(3) The intensity of selected titanium nitride reflections is a function of the tilting angle $\vartheta$ or $\Omega$.

For determination of the ODF $f(\vec{h})$ of the fibre texture the strongest diffraction rings of $\delta$ - $\mathrm{TiN}_{x}$ were chosen. In Fig. 2 the normalized intensities for the four reflections (10ī0)-Ti, (10i1)-Ti, (111)-Ti, and (200)-TiN are illustrated as function of the tilt angle $\vartheta$ in $10^{\circ}$-steps after perpendicular implantation. The dominant peaks are Ti reflections. Partly, the titanium nitride reflection could not be identified (e.g. the (111)-TiN peak for $\Omega>40^{\circ}$ ). In Fig. 3 are shown the corresponding incomplete pole figures $\left(30^{\circ} \leq \vartheta \leq 90^{\circ}\right)$ of the analy sed four $\delta$ - $\operatorname{TiN}_{x}$ reflections and also the normalized intensity of the transmitted primary electrons as function of the tilt angle for the whole range of tilting after nitrogen ion implantation at room temperature. The ODF was determined with a modified WIMV-method ${ }^{4}$ on the basis of the (200), (220) and (111) pole figures. The reproduced pole figures are shown Fig. 4. The associated ODF is exhibited in Fig. 5 . The main component (111) $\| \vec{N}$ or (111) $\| \vec{C}$ is remarkable in contrast to the randon distribution (about 12 times).

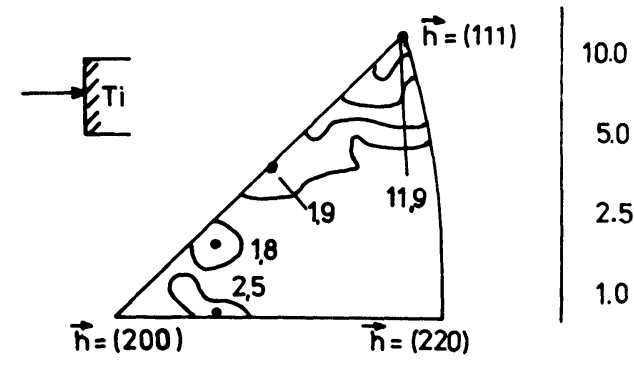

Figure 5: Orientation distribution of normals of net planes $\vec{h}_{i} \| \vec{N}$ of TiN after implantation perpendicular to the surface

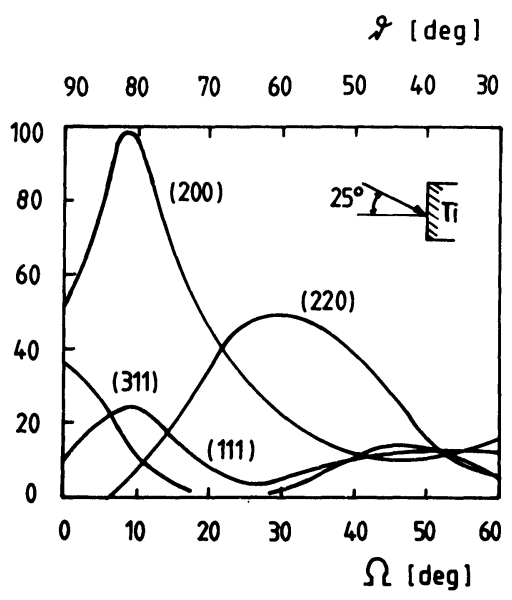

Figure 6: Diffraction pole figures $(200),(111),(220)$ and $(311)$. The implantation direction was $25^{\circ}$ from the normal incidence.

\section{Discussion}

The preferred orientation or pronounced alignment effect by ion implantation was differently interpreted ${ }^{4,9}$ :

- The effect may be due to the strong compressive strains induced by highfluence implantation ${ }^{12}$. These strains can causes a slip mechanism ${ }^{6}$ or a polygonization process ${ }^{14}$. 
- On the other side, the alignment effect was explained by the so-called seed selection process through ion channeling ${ }^{7,8}$, i.e. the implanted ions should destroy a major fraction of grains and preserve only grains of crystallographic orientations where the lattice damages can be minimized by ion channeling (e.g. the [111] direction of titanium nitride precipitates).

In order to investigate the causes of the formation of the restricted-fibre textured polycrystalline thin film, we choose an angle of incidence nitrogen ions so that the ion beam selected growth direction is changed. But nevertheless, the strong compressive strain in the near-surface range is approximately unchanged. On this basis it should be possible to distinguish between the different causes of the implantation-induced texture. By choosing the angle of ion implantation of $25^{\circ}$ from the normal incidence, the ion beam should select the main axial channeling direction of incidence, i.e. the fibre axis should be changed by about $25^{\circ}$. If the induced strain dominates, the fibre axis is unchanged and the (111) plane should be always abserved perpendicular to the surface.

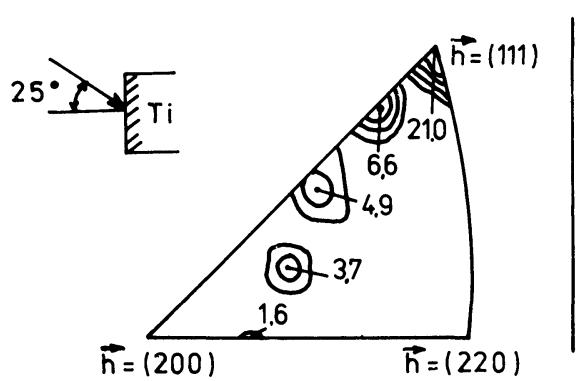

Figure 7: Orientation distribution of normals of net planes $\vec{h}_{1} \| \vec{C}$ of TiN. The implantation direction was $25^{\circ}$ from the normal incidence.

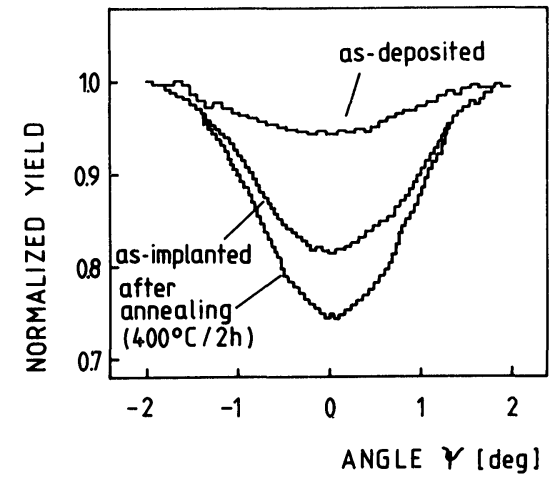

Figure 8: Angular yield curves be-

fore implantation (as-deposited), after implantation and after annealing

In Fig. 6 the pole figures of the four reflections

$(200),(111),(220)$ and (311) TiN are shown as a function of the tilt angle $\vartheta$ in $10^{\circ}$-steps after implantation under an angle $25^{\circ}$ from the normal incidence $\left(25^{\circ}\right.$-implantation). In contrast to the intensities of the titanium nitride reflections in Fig. 3, we found modified intensities. For example, the maximum of the (200) peak was found at $55^{\circ}$ after perpendicular implantation and near $80^{\circ}$ after $25^{\circ}$ - implantation i.e. the peak maximum is shifted by about $25^{\circ}$. On the basis of these measurements, the ODF (cf. Fig. 7) was determined, where the fibre axsis $\vec{C}$ corresponds to the direction of implantation. Also in this case, the (111) component is the main-component $[(111) \| \vec{C}]$ and the positions of the sub-maximum, e.g. (220) and (200), are also comparable. This is in a good agreement with the results after perpendicular implantation (cf. Fig. 5).

In addition, the technique of ion channeling ${ }^{15}$ was used to prove the channeling direction in the implantation induced titanium nitride. Angular yield 
curves for $\mathrm{Ti}$ before and after implantation with $5 \times 10^{17} \mathrm{~N}^{+}$-ions $/ \mathrm{cm}^{2}$ are demonstrated in Fig. 8 . With respect to the polycrystalline structure of the thin films, the minimum of the normalized field $(\approx 0.85 \pm 0.05)$ can be interpreted as a channeling direction. An annealing process at $400^{\circ} \mathrm{C}$ for $2 \mathrm{~h}$ in a high vacuum was subsequently carried out to enhance this effect. As pictured in Fig. 8 , the minimum of the normalized yield is decreased $(\approx 0.77 \pm 0.05)$ and the average grain size is increased from the above-mentioned $100 \mathrm{~nm}$ to about 250 $\mathrm{nm}$. The detailed angular scan measurements, recording the anglar dependence of the normized yields, demonstrate that a strongly channeling axsis is formed by high-fluence ion implantation. With the help of the outcome of the electron diffractions experiments, it is possible to identify this channeling axsis with the [111] TiN-direction, i.e. with the fibre axsis $\vec{C}$.

The results of electron diffraction and ion channeling measurements show that the normal of the (111) plane is strongly coupled with the direction of implantation. As shown in Fig. 5 and Fig. 7, this coupling with the implantation direction was also found for the sub-maximum of minor strength.

In summary, the results indicate that a controlled modification of the texture of implantation-induced precipitates is possible by high-fluence ion implantation. This allows the possibility of modification of the anisotropic properties of polycrystalline material, for example in respect to an improvement of wear or corrosion resistance.

\section{REFERENCES}

1. F. Haessner, K. Jakubowski and M. Wilkens : Acta Metall. 14454 (1966)

H. Perlwitz, K. L"ucke and W. Pietsch : Acta Metall. 171183 (1964)

2. R. Schwarzer :Z. Metallkde. 73495 (1982)

3. F.J. Humphrey :Textures Microstr. 645 (1983)

4. K. Helming and B. Rauschenbach :Phys. Status Solidi (b) 138 K1 (1986)

5. B. Rauschenbach and K. Helming : Nucl. Instr. and Meth. 42216 (1989)

6. O. Meyer and A. Azzam :Phys. Rev. Lett. 521629 (1984)

7. K.T.-Y. Kung and R. Reif :J. Appl. Phys. 592422 (1986)

8. J.M.E. Harper, D.A. Smith, L.S. Yu and J.J. Cuomo:Beam-Solid and Phase Transformation, eds. H. Kurz, G.L. Olsen and J.M. Poate : Mater. Res. Soc. Symp. Proc. 51127 (1985)

9. K. Hohmuth and B. Rauschenbach :Mater. Sci. Engng. 69489 (1985)

10. S. Ohra and M. Iwaki :Nucl. Instr. and Meth. B19/20 162 (1987)

11. G. Marest : in Ion Implantation ed. F.H. Wöhlbier, Trans Tech Publ. Ltd., Aedermannsdorf, 273 (1988)

12. S. Fayeulle and D. Treheux : Nucl. Instr. and Meth. B19/20 216 (1987)

13. B. Rauschenbach: J. Mater. Sci. 21395 (1985)

14. M. Gettings, O. Meyer and G. Linker:Radiat. Eff. 2151 (1974)

15. D.S. Gemmell:Rev. Mod. Phys. 46129 (1974) 